\title{
Performance Evaluation of a Regenerative Pump under Fluid Viscosity Enhancement
}

\author{
J. NEJADRAJABALI*, A. RIASI**, S. A. NOURBAKHSH*** \\ *University of Tehran, School of Mechanical Engineering, Tehran, Iran, E-mail: J.nejad@ut.ac.ir \\ **University of Tehran, School of Mechanical Engineering, Tehran, Iran, E-mail: ariasi@ut.ac.ir \\ ***University of Tehran, School of Mechanical Engineering, Tehran, Iran, E-mail: anour@ut.ac.ir \\ cross $^{\text {ref }}$ http://dx.doi.org/10.5755/j01.mech.23.6.16204
}

\section{Introduction}

Regenerative flow pumps are types of dynamic pumps with the ability to develop high heads at low flow rates. So they have very low specific speed and can replace the positive displacement pumps without any wear and lubrication problems. Furthermore, RFPs are capable of working in low NPSH. Therefore, Regenerative Pumps are better choice than other types of centrifugal pumps at low specific speeds [1]. Regenerative pumps capable of generating heads equivalent to that of several centrifugal stages with comparable tip speeds. RFPs have compact design and they are very cheap to manufacture. They present performance curve with very stable features [2]. Despite the disadvantage of having low hydraulic efficiency (30-50\%), regenerative pumps have found many applications in industries including automotive and aerospace fuel pumping, booster systems, water supply, agricultural industries, shipping and mining, chemical, and food processing systems [3].
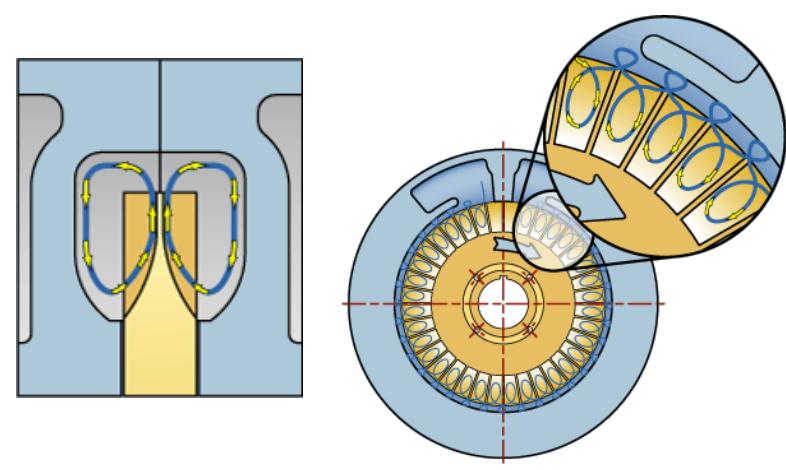

Fig. 1 Cross sections of casing and flow channel, showing helical flow pattern [4]

Regenerative pumps use a free rotating type impeller, like other types of turbo- pumps. Impeller has blades machined into each side at its periphery. As seen in Fig. 1, the fluid moves spirally in flow channel and reenters the blades of impeller several times in its peripheral path from inlet to outlet which forms a torroidal motion. The Fluid enters the flow channel with inlet port and discharges with high pressure from the outlet port. The stripper occupies the space between inlet and outlet regions of casing to separate low-pressure inlet from high-pressure outlet. The stripper helps the fluid to go out from discharge port. Just the fluid within impeller blades can pass through stripper.

The idea of regenerative flow pump was present- ed by Adolph Wahle and the first model of it was manufactured by Western Pump company at 1918 [5]. Despite existence of several experimental and theoretical analyzes about regenerative flow pump, the number of articles in this field is low compared to other types of turbomachines.

Bartles attempted to study the flow mechanism in regenerative pumps experimentally using three different rotors with the same flow channel. He found that the pump would only work with the impeller which was grooved out and allowing circulatory motion and centrifugal pumping [4]. Lazo and Hopkins setup an experimental test rig for a regenerative pump. They used small thread probes to measure and observe flow velocities and angles in flow passage of the pump to obtain better information for flow pattern recognition; at the same time Lutz investigated the pressure distribution from inlet to outlet of the regenerative pump [3]. Crewdson studied the circulatory flow between blades and flow channel of regenerative pump. He divided the flow channel in to two parts by soldering a thin brass strip along the middle of it. So the circulatory flow was affected. He concluded that cluttering the circulatory flow pattern would reduce the head and efficiency of the pump [4]. The effect of non-radial blades on regenerative pump performance was investigated by Burton. He found that using a $47^{\circ}$ blade angle would increase the head about twice of that obtained by radial blades at shut-off point [6]. A new model for regenerative pump was presented by Badami, in which both inlet and outlet ports were designed axially. He also studied some geometric parameters of regenerative pumps and used the momentum exchange theory proposed by Wilson et al. to present a comparison between experimental and theoretical data [7]. Engeda reviewed the status of regenerative pumps and proposed a Modified model which could describe the change in rotation due to variation in channel region. Predicted results of the model were compared with experimental data [8]. Yoo et al. studied the design of a regenerative flow pump for artificial heart pump application based on an improved momentum exchange theory which could calculate the geometry of rotating flows $[9,10]$. Quil et al. used a commercial code to investigate the fluid flow in a regenerative pump. They also carried out a new method of manufacturing to evaluate the influence of change in blade geometry on the efficiency of regenerative pump $[11,12]$. An experimental study was accomplished by Choi et al. to investigate the effect of the impeller blade angle and its shape on regenerative pump performance. Among all blade configurations tested in this study, the chevron blade exhibited the highest head with reasonably good efficiency. It was found 
that there was an optimum chevron angle of around $30^{\circ}$ [13]. Fleder and Böhle studied the effect of the variation of the blade length, blade width, and side channel height by the help of dimensionless parameters [14]. Karanth et al. used CFD analysis to enhance the performance of regenerative pump. They found that the number of blades and the inlet and outlet passage of the regenerative pump have significant effect on the pump performance [15]. Maity et al. also used CFD to study regenerative pumps. They found that a curvature in the outlet flow domain minimizes the vortex flow, so the net pressure head increases. Also, positioning of the blades on either side of the impeller by offsetting enhances the fluid motion and results into the net increase in static and net pressure head [16]. Karlsen and Aggidis offered an extensive review into the development, performance challenges and design improvements of Regenerative pumps with a particular focus on improving efficiency throughout the pump life cycle [4]. Nejadrajabali et al. carried out a numerical study to investigate the effect of blade profile on the performance of regenerative pump [17].

The above literature indicates that the effect of fluid viscosity on the performance of regenerative pumps has not been the focus of studies and hence an attempt has been made in this paper to investigate experimentally the influence of fluid viscosity on the performance of regenerative pump. The primary fluid was water with viscosity of 1 centipoise. Polyacrylamide (PAM) which is a soluble polymer in water was employed to increase the viscosity of fluid. The mixture of water and PAM was pumped several times for breaking or shearing its molecules at constant temperature to have a homogenous fluid. A Brookfield viscometer was used for measuring viscosity. Solutions prepared from dry powder PAM at two concentrations of $1600 \mathrm{ppm}$ and $2400 \mathrm{ppm}$. The measured viscosities for these two solutions were 180 and 300 centipoises respectively. The performance curves of the regenerative pump working with these fluids were compared to assess the effect of fluid viscosity on the operation of regenerative pump.

\section{Specifics of regenerative pump}

Fig. 2 shows the components of the regenerative pump consists of an impeller with radial blades at its periphery, inlet and discharge port, stripper to isolate the high-pressure discharge from the low-pressure inlet, flow passage and a casing. The main geometric parameters of the tested regenerative pump are presented in Table 1 and also in Fig. 3. The radial blades impeller for regenerative pump is shown in Fig. 4.

Table 1

Geometric specifications of regenerative pump

\begin{tabular}{|l|c|c|}
\hline \multicolumn{1}{|c|}{ Parameters } & unit & Value \\
\hline Number of blades in each sides, $N$ & --- & 36 \\
\hline Tip radius of casing, $r_{S}$ & $\mathrm{~mm}$ & 36 \\
\hline Tip radius of blade, $r_{T}$ & $\mathrm{~mm}$ & 32.5 \\
\hline Hub radius of blade, $r_{H}$ & $\mathrm{~mm}$ & 23.5 \\
\hline Height of blade, $h$ & $\mathrm{~mm}$ & 9 \\
\hline Width of the blade, $b$ & $\mathrm{~mm}$ & 3.1 \\
\hline Width of the channel, $d$ & $\mathrm{~mm}$ & 4.3 \\
\hline Angle of stripper, $\theta_{s}$ & ${ }^{\circ}$ & 16 \\
\hline
\end{tabular}

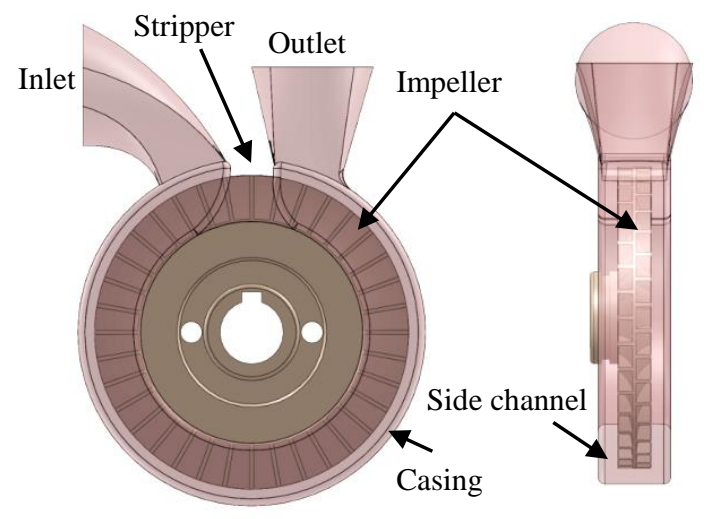

Fig. 2 Schematic of the regenerative pump

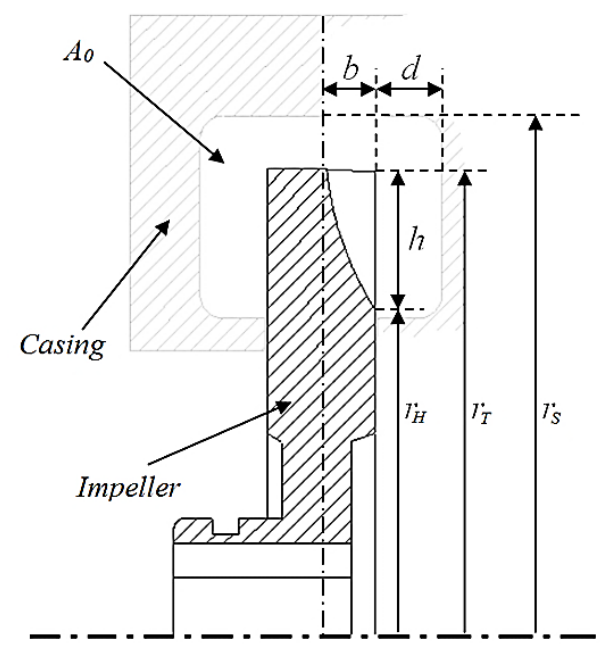

Fig. 3 Regenerative pump geometric parameters

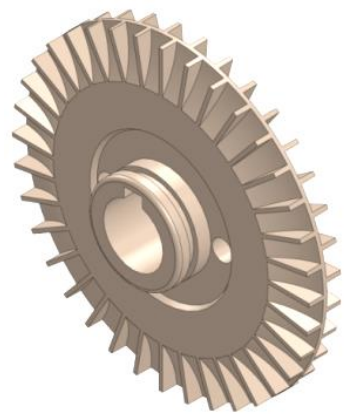

Fig. 4 Radial blades impeller for regenerative pump

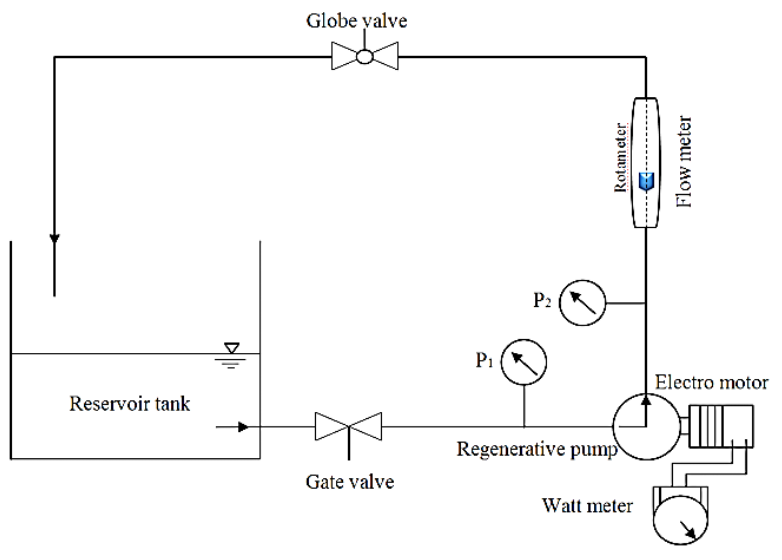

Fig. 5 Schematic of regenerative pump test rig 


\section{Experiments apparatus}

The arrangement of test rig is indicated in Fig. 5. As it can be seen a reservoir tank with the capacity of 150 liters was employed to store and ultimately receive fluid. For flow rate adjustment, a control valve was installed in the return line to the reservoir tank. The flow rate was measured using a rotameter which was calibrated.

The pump was driven by a 500-watt induction motor operating at a constant speed of $2900 \mathrm{rpm}$. For power consumption of the electro pump, a watt meter with the accuracy of about $\pm 1.5 \%$ was employed. Fig. 6 represents the performance curve of the electromotor. It was obtained using a dynamometer, separately. So the power on the shaft can be obtained to use in the calculation of pump hydraulic efficiency by Eq. (1).

$$
P_{2}=P_{1} \times \eta_{e}
$$

where $P_{1}$ is power consumption of electromotor and $P_{2}$ is Power on the shaft.

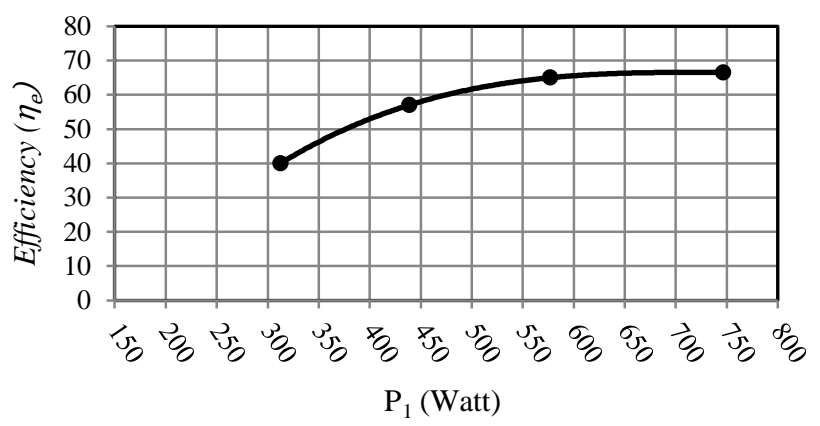

Fig. 6 Performance curve of electromotor (efficiency versus power consumption)

A proximity sensor was used to measure the rotational speed. The inlet and outlet pressures were measured using two analog pressure gauges.

Viscosity measurement of working fluids was carried out with a Brookfield viscometer. The schematic of viscometer has been shown in Fig. 7. The principle of operation of this kind of viscometer is to rotate a spindle immersed in the fluid and measuring the viscous drag of the test fluid against the spindle through the deflection of a calibrated spring. The deflection of spring is diagnosed with a rotary transducer. The viscometer has full scale range accuracy within $\pm 1 \%$.

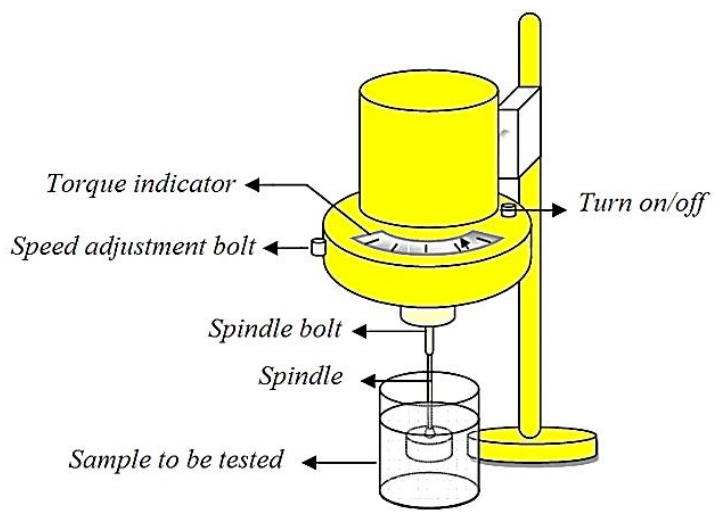

Fig. 7 Schematic of viscometer and its components
The following equations (equations 2-5) are used for drawing curves of shear stress and viscosity of fluid versus shear rate.

$$
\begin{aligned}
& \tau_{i}=\frac{2 T}{\pi D_{i}^{2} l}, \\
& {\left[\frac{d u}{d r}\right]_{i}=\frac{4 \pi N_{s p}}{n^{\prime \prime}},} \\
& n^{\prime \prime}=\text { Slope of } \frac{d \ln (T)}{d \ln \left(N_{s p}\right)}, \\
& \mu=\frac{\tau_{i}}{\left[\frac{d u}{d r}\right]_{i}},
\end{aligned}
$$

where $\tau_{i}$ is shear stress, $D_{i}$ is diameter of spindle, $l$ is effective length of spindle and $N_{s p}$ is the rotational speed of spindle.

Polyacrylamide (PAM) which is a soluble polymer in water was employed to increase the viscosity of water, see Fig. 8. The technical specifications for Polyacrylamide (grade A500) are presented in table 2. Solutions prepared from dry powder Polyacrylamide (PAM) at two concentrations of $1600 \mathrm{ppm}$ and $2400 \mathrm{ppm}$. According to experimental studies of Bjorneberg, the viscosity of PAM solution reduces by pumping. The viscosity reduction is thought to result from breaking or shearing the PAM molecules. He reported that pumping a 2400 ppm PAM solution just once through a centrifugal pump reduces viscosity 15 to $20 \%$; pumping five times reduces viscosity approximately $50 \%$. But there was not significant change in viscosity of PAM solution through pumping more than five times [18]. So it shows that to have a homogeneous solution it is necessary to pump PAM solutions several times.

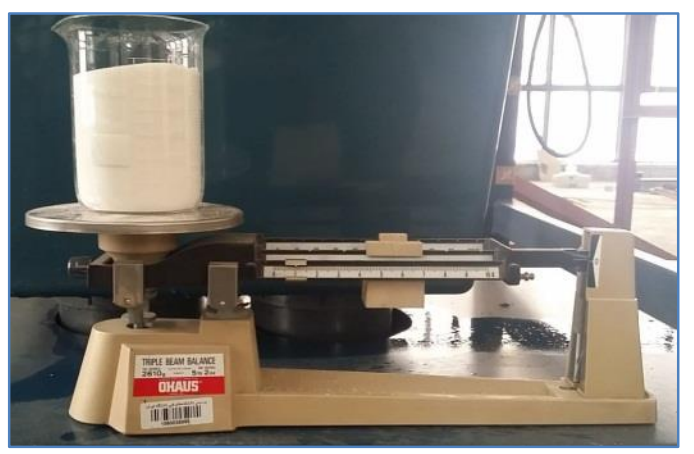

Fig. 8 Polyacrylamide grade A500 being weighting

Technical specification of PAM

Table 2

\begin{tabular}{|c|l|l|}
\hline \multicolumn{3}{|c|}{ Polyacrylamide (grade A500) - medium molecular weight } \\
\hline Item & Description & Result of test \\
\hline 1 & Chemical Name & Polyacrylamide - Polyelectrolyte \\
\hline 2 & Ionic action & Anionic \\
\hline 3 & CAS Number & $9003-05-8$ \\
\hline 4 & Water soluble & Fast \& complete \\
\hline 5 & Appearance & White Granule \\
\hline
\end{tabular}




\section{Testing procedures}

As mentioned, the main objective of this experimental work is to investigate the effect of viscosity change on the performance of regenerative pumps. Thus, as summarized in Table 3, three fluids with different viscosities were employed. In this study the working fluid was pumped more than 30 times to have a homogeneous solution in each case. Then, a sample was taken for measuring viscosity by Brookfield viscometer as seen in Fig. 9.

Table 3

Specifications of working fluids

\begin{tabular}{|l|c|c|c|}
\hline $\begin{array}{c}\text { Working } \\
\text { fluid }\end{array}$ & Concentration & $\begin{array}{c}\text { Dynamic vis- } \\
\text { cosity }\end{array}$ & $R_{T}$ \\
\hline Water & ---- & 1 centipoise & $3.8 \times 10^{7}$ \\
\hline Solution 1 & $1600 \mathrm{ppm}$ & 180 centipoise & $2.1 \times 10^{5}$ \\
\hline Solution 2 & $2400 \mathrm{ppm}$ & 300 centipoise & $1.3 \times 10^{5}$ \\
\hline
\end{tabular}

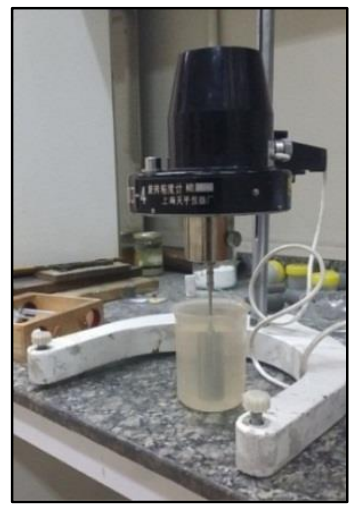

Fig. 9 Polyacrylamide grade A500 being weighting

For each working fluid, the pressures and power consumption were measured with controlling the flow rate by the flow control valve. The operating conditions are presented in Table 4 and the test rig arrangement is shown in Fig. 10.

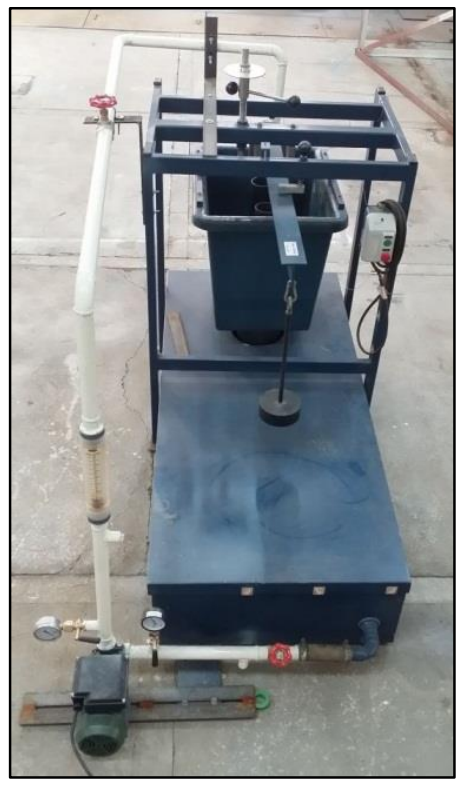

Fig. 10 Test rig arrangement
Experiments operating conditions

Table 4

\begin{tabular}{|l|c|c|}
\hline Parameters & Unit & Value \\
\hline Pressure & $\mathrm{kpa}$ & 86.2 \\
\hline Temperature & ${ }^{\circ} \mathrm{C}$ & 22.6 \\
\hline Rotational speed & $\mathrm{rpm}$ & 2900 \\
\hline
\end{tabular}

\section{Results and discussions}

Regenerative flow pumps confirm affinity laws as centrifugal and axial pumps. So the experimental measurements of the regenerative pump performance characteristics are expressed using dimensionless parameters. The flow coefficient $(\phi)$, head coefficient $(\psi)$, power coefficient $(\tau)$ and hydraulic Efficiency $(\eta)$ which is defined as the ratio between the hydraulic power transferred to the working fluid and the mechanical power introduced into the system by the impeller [13]. Pump characteristic flow, head, power and efficiency coefficients can be expressed as follow:

$$
\begin{aligned}
& \psi=\frac{g H}{U_{g}^{2}}, \\
& \phi=\frac{Q}{A_{0} U_{g}}, \\
& \tau=\frac{P}{\rho A_{0} U_{g}^{3}}, \\
& \eta=\frac{\rho Q g H}{P}=\frac{\phi \psi}{\tau},
\end{aligned}
$$

where $U_{g}$ is tangential velocity, $A_{0}$ is the cross section area of channel and $\rho$ is the density of working fluid.

Fig. 11 shows the characteristic curves of the regenerative pump, using water as working fluid. As it can be seen the maximum hydraulic efficiency occurs at $\phi_{n}=$ 0.47 with about $42.5 \%$. At shut-off condition $(\phi=0)$, the head coefficient $(\psi)$ is about 5.3 and the power coefficient $(\tau)$ is obtained about 4.4 .

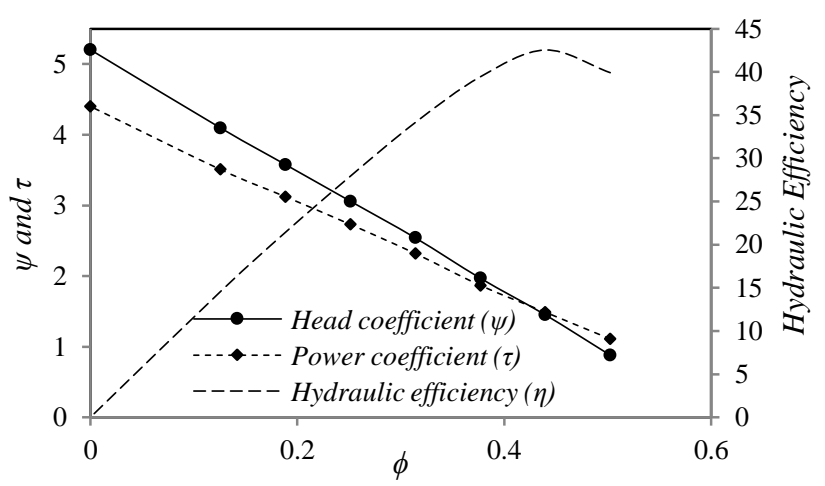

Fig. 11 Characteristic curves of pump using water as working fluid

Fig. 12 indicates the characteristic curves of the regenerative pump at three different viscosities of working 
fluid. The primary fluid was water with the viscosity of 1 centipoise. As mentioned, a soluble polymer (Polyacrylamide) in water was used to change the viscosity of fluid. Solutions prepared from dry powder PAM at two concentrations of $1600 \mathrm{ppm}$ and $2400 \mathrm{ppm}$. To have a homogeneous fluid, the solutions were pumped more than 30 times. A Brookfield viscometer was used for measuring the viscosities of solutions at these two concentrations. Viscosities for these two solutions were 180 and 300 centipoises, respectively.

As it can be seen from Fig. 12, a, by increasing the viscosity of working fluid the head coefficient reduces. It is observed that for higher flow coefficients (more than $0.5)$, there were not significant changes in head coefficients. But as flow coefficient reduces, the decline in head coefficient gradually increases. The percent of reduction in head coefficient at shut-off point $(\phi=0)$, for the pump working with solution 1 (180 centipoise) and solution 2 (300 centipoise), relative to that of working with water, is $43.4 \%$ and $67 \%$, respectively.

Fig. 12, b shows the variations of power coefficient in terms of flow coefficient. Trends of these curves are similar to head coefficient. Viscosity enhancement reduces the power coefficient. The reduction in power is significant at lower flow rates. The amount of power coefficient at shut-off point is reduced about $50 \%$ by changing viscosity from 1 to $180 \mathrm{cp}$. The value of power coefficient at shut-off condition for regenerative pump working with solution 2 (300 centipoise) is about 1.8 which is $59 \%$ lower than the power coefficient of the pump working with water.

As summarized in Table 3, the impeller tip speed Reynolds number $\left(R_{T}\right)$ represented by equation 10 reduces by increasing the viscosity of fluid. Thus, with reduction in Reynolds number, the regenerative pump characteristics deteriorate.

$$
R_{T}=\frac{U_{g} D}{v}
$$

where $v$ represents kinematic viscosity.

The comparison of hydraulic efficiency curves for these three cases is indicated in Fig. 12, c. It was not observed any significant changes in efficiency curves at low flow coefficients. It because the more the head coefficient reduces the more the power coefficient decreases. But around design condition or in other words, around the best efficiency point, the hydraulic efficiency of the pump reduces by increasing viscosity of working fluid. At best efficiency point $\left(\phi_{n}=0.47\right)$, the efficiency of the pump working with solution $1(180 \mathrm{cp})$ is about $40.2 \%$ which is $2.3 \%$ lower than the efficiency of the pump uses water as working fluid. The efficiency of the pump working with solution $2(300 \mathrm{cp})$ is about $33.7 \%$ which is $8.8 \%$ lower than the pump working with water $(1 \mathrm{cp})$. Eventually, it was found that increasing the viscosity of working fluid would reduce the performance characteristics of the regenerative pump. So, it seems that regenerative pumps are not good choices for pumping fluids with very high viscosities.
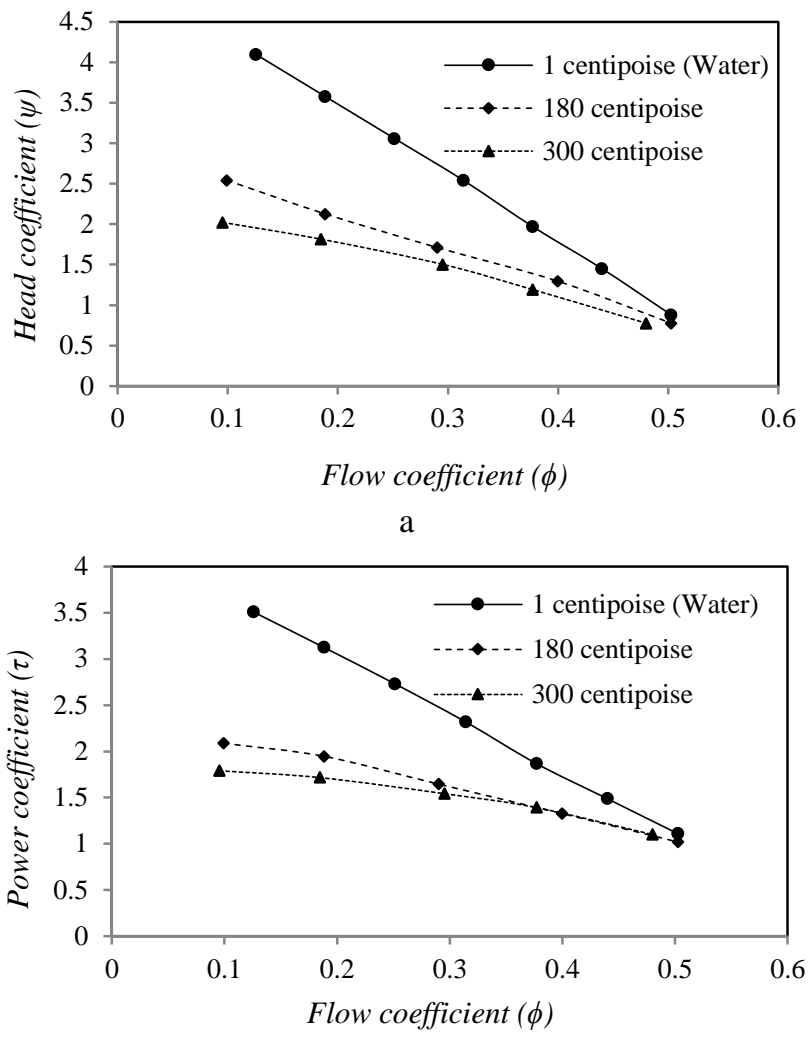

$\mathrm{b}$

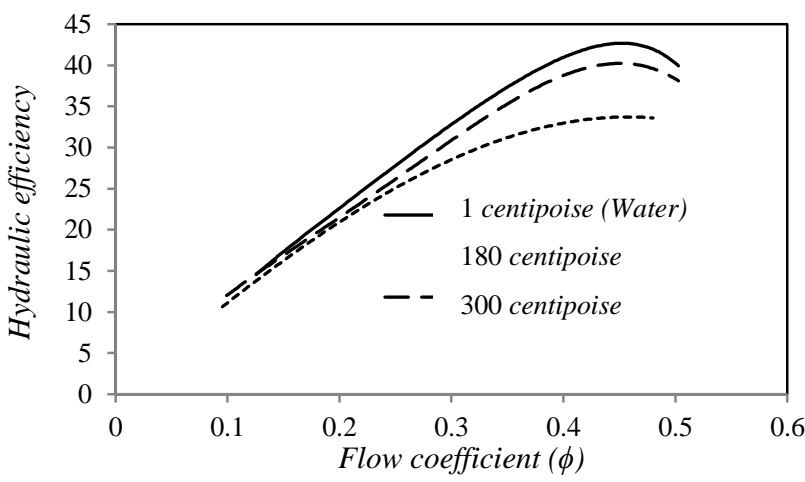

c

Fig. 12 Characteristic curves of regenerative pump at different viscosity fluids. a) Head coefficient versus flow coefficient. b) Power coefficient versus flow coefficient. c) Hydraulic efficiency versus flow coefficient

\section{Uncertainty analysis}

Considering the output quantity denoted by $R$, is related to $n$ variables as:

$$
R=R\left(x_{1}, x_{2}, \ldots, x_{n}\right) .
$$

The overall uncertainty, $W_{R}$ will obtain from relation (12):

$$
W_{R}=\left[\left(\frac{\partial R}{\partial x_{1}} w_{1}\right)^{2}+\left(\frac{\partial R}{\partial x_{2}} w_{2}\right)^{2}+\ldots+\left(\frac{\partial R}{\partial x_{n}} w_{n}\right)^{2}\right],
$$

where $w_{n}$ is the associated uncertainty of independent measurement $x_{n}$. 
From equation $13, \Delta P, Q$ and $\Delta Z$ are the independent variables for calculating the pump head.

$$
\Delta H=\frac{\Delta P}{\rho g}+\frac{V_{2}^{2}-V_{1}^{2}}{2 g}+\Delta Z .
$$

The uncertainty in the measurement of flow rate was about $\pm 2 \%$. And the pressures were measured with the uncertainty of $\pm 1.5 \%$. The uncertainty for $\Delta Z$ is about $\pm 0.1 \%$. The results of uncertainty analysis are tabulated in Table 5. The average of uncertainties is about 3\% which indicates the acceptability of test results.

Table 5

Uncertainty analysis

\begin{tabular}{|l|l|c|c|c|c|c|c|c|c|}
\hline \multirow{4}{*}{$\begin{array}{l}\text { Water } \\
\text { (1 centipoise) }\end{array}$} & Flow rate (lit/min) & 0 & 10 & 15 & 20 & 25 & 30 & 35 & 40 \\
\cline { 2 - 10 } & Head (meter) & 54.1 & 40.7 & 35.5 & 30.4 & 25.2 & 19.6 & 14.4 & 8.8 \\
\cline { 2 - 10 } & Uncertainty (meter) & 1.622 & 1.220 & 1.066 & 0.911 & 0.757 & 0.587 & 0.432 & 0.263 \\
\cline { 2 - 10 } & Uncertainty (\%) & 2.998 & 2.998 & 3.002 & 2.998 & 3.003 & 2.994 & 3.003 & 2.984 \\
\hline \multirow{4}{*}{$\begin{array}{l}\text { Solution 1 } \\
(180 \text { centipoise) }\end{array}$} & Flow rate (lit/min) & 8 & 15 & 23 & 32 & 40 & & & \\
& Head (meter) & 25.2 & 21.1 & 17.0 & 12.9 & 7.7 & & & \\
\cline { 2 - 10 } & Uncertainty & 0.757 & 0.633 & 0.510 & 0.386 & 0.232 & & & \\
\cline { 2 - 9 } & Uncertainty (\%) & 3.003 & 3.001 & 2.998 & 2.993 & 3.009 & & & \\
\hline \multirow{4}{*}{$\begin{array}{l}\text { Solution 2 } \\
\text { (300 centipoise) }\end{array}$} & Flow rate (lit/min) & 7.5 & 15 & 23.5 & 30 & 38 & & & \\
\cline { 2 - 10 } & Head (meter) & 20.1 & 18.0 & 14.9 & 11.8 & 7.7 & & & \\
\cline { 2 - 9 } & Uncertainty & 0.602 & 0.541 & 0.448 & 0.355 & 0.232 & & & \\
\cline { 2 - 9 } & Uncertainty (\%) & 2.997 & 3.003 & 3.006 & 3.010 & 3.009 & & & \\
\hline
\end{tabular}

\section{Conclusions}

In this study, the influence of fluid viscosity on the performance of regenerative pump was investigated experimentally. The primary fluid was water with viscosity of 1 centipoise. A soluble polymer in water named polyacrylamide (PAM) was used to change the viscosity of fluid. The working fluids were pumped more than 30 times to have homogeneous solutions in each case. A Brookfield viscometer was employed for measuring viscosity. Solutions prepared from dry powder PAM at two concentrations of $1600 \mathrm{ppm}$ and $2400 \mathrm{ppm}$. The measured viscosties for these two solutions were 180 centipoises (solution 1) and 300 centipoises (solution 2) respectively. Results showed that increase in the viscosity of working fluid would decrease the head coefficient. It is observed that as flow coefficient reduces, the decline in head coefficient gradually increases. It was found that viscosity enhancement reduces the power coefficient. Similar to head coefficient, the reduction in power is considerable at lower flow rates. According to the comparison of the hydraulic efficiency curves, there was not any significant change in efficiency curves at low flow coefficients. It because the more the head coefficient reduces the more the power coefficient decreases due to enhancement in viscosity of working fluid. Contrary to low flow coefficients, around design flow coefficient and high flow coefficients, the hydraulic efficiency of the pump reduces by increasing viscosity of working fluid. The results of this experimental study showed that viscosity enhancement would reduce the performance characteristics of regenerative pumps. Thus, it could be concluded that regenerative pumps are not suitable for pumping fluids with very high viscosities or in other words regenerative pumps are not good choice in low Reynolds numbers.

\section{Acknowledgment}

The authors acknowledge the support of Iranian Gas Transmission Co.

\section{References}

1. Muller, S. 2004. Consider regenerative pumps for low flow/low NPSH applications, Hydrocarbon Process (Special Report), 55-57. www.HydrocarbonProcessing.com.

2. Raheel, M.; Engeda, A. 2005. Systematic design approach for radial blade regenerative turbomachines, Propulsion and Power 21(5): 884-892. http://dx.doi.org/10.2514/1.1426.

3. Raheel, M.; Engeda, A. 2002. Current status, design and performance trends for the regenerative flow compressors and pumps, Proceedings of IMECE.

4. Karlsen-Davies, N.D.; Aggidis, G.A. 2016. Regenerative liquid ring pumps review and advances on design and performance, Applied Energy 164: 815-825. http://dx.doi.org/10.1016/j.apenergy.2015.12.041.

5. Mowrey, C.C. 1987. High Head - Low Flow Centrifugal Pumps, ASLE Annual Meeting, Colorado, 155-161.

6. Burton, D.W. 1962. Review of Regenerative Compressor Theory., Rotating Machinery for Gas-Cooled Reactor Application, TID-7631, April 2-4, 228-242. http://dx.doi.org/10.1115/IMECE2002-39594.

7. Badami, M. 1997. Theoretical and Experimental Analysis of Traditional and New Periphery Pumps, SAE Technical paper, Series (971074): 45-55. http://dx.doi.org/10.4271/971074.

8. Engeda, A. 2003. Flow Analysis and Design Suggestions for Regenerative Flow Pumps, Proceedings of ASME FEDSM'03, 4th ASME JSME Joint Fluids Engineering Conference.

9. Yoo, I.S.; Park, M.R. and Chung, M.K. 2006. Hydraulic design of a regenerative flow pump for an artificial heart pump, IMechE 220: 699-706. http://dx.doi.org/10.1243/09576509JPE211.

10. Yoo, I.S.; Park, M.R. and Chung, M.K. 2005. Improved momentum exchange theory for incompressible regenerative turbomachines, Proc. Inst. Mech. Eng. Part A J. Power Energy 219: 567-581. http://dx.doi.org/10.1243/095765005X31252.

11. Quail, F.; Scanlon, TH.; Stickland, M. 2011. Design 
optimisation of a regenerative pump using numerical and experimental techniques, International Journal of Numerical Methods for Heat \& Fluid Flow 21(1): 95111. http://dx.doi.org/10.1108/09615531111095094.

12. Quail, F.; Scanlon, TH.; Baumgartner, A. 2012. Design study of a regenerative pump using onedimensional and three-dimensional techniques, European Journal of Mechanics B/Fluids 31: 181-187. http://dx.doi.org/10.1016/j.euromechflu.2011.06.003.

13. Choi, C.W.; Yoo, S.I.; Park, R.M. and Chung, K.M. 2013. Experimental study on the effect of blade angle on regenerative pump performance, Proc IMechE Part A: Journal of Power and Energy. http://dx.doi.org/10.1177/0957650913487731.

14.Fleder, A.; Böhle, M. 2015. A Systematical Study of the Influence of Blade Length, Blade Width, and Side Channel Height on the Performance of a Side Channel Pump, ASME Journal of Fluids Engineering, 137(12), p. 121102. http://dx.doi.org/10.1115/1.4030897.

15. Vasudeva Karanth, K.; Manjunath, M. S.; Kumar, S. and Yagnesh Sharma, N. 2015. Numerical Study of a Selfpriming Regenerative Pump for Improved Performance using Geometric Modifications, International Journal of Current Engineering and Technology 5(1): 104-109.

Available at http://inpressco.com/category/ijcet.

16. Maity, A.; Chandrashekharan, V. and Afzal, M.W. 2015. Experimental and Numerical Investigation of Regenerative Centrifugal Pump using CFD for Performance Enhancement, International Journal of Current Engineering and Technology 5(4): 2898-2903. Available at http://inpressco.com/category/ijcet.

17. Nejadrajabali, J.; Riasi, A. and Nourbakhsh, S.A. 2016. Flow Pattern Analysis and Performance Improvement of Regenerative Flow Pump Using Blade Geometry Modification, International Journal of Rotating Machinery, Volume 2016.

http://dx.doi.org/10.1155/2016/8628467.

18. Bjorneberg, D. L. 1998. Temperature, Concentration, and Pumping effects on PAM viscosity, Transactions of the ASAE 41(6): 1651-1655.

https://doi.org/10.13031/2013.17340.

J. Nejadrajabali, A. Riasi, S. A. Nourbakhsh

\section{PERFORMANC EVALUATION OF A REGENRATIVE PUMP UNDER FLUID VISCOSITY ENHANCEMENT}

S u m m a r y

Regenerative flow pumps are kinds of dynamic pumps with the ability to develop high heads at low flow rates. Despite the disadvantage of having low hydraulic efficiency, regenerative flow pumps have found many applications in industries. In this paper, the effect of fluid viscosity on the performance of regenerative pump has been investigated experimentally. The primary fluid was water with viscosity of 1 centipoise. Polyacrylamide (PAM) which is a soluble polymer in water was used to increase the viscosity of fluid. Solutions prepared from dry powder PAM at two concentrations of $1600 \mathrm{ppm}$ and 2400 ppm. Solutions were pumped more than 30 times to have homogeneous fluids. The measured viscosities for these two solutions were 180 and 300 centipoises respectively. Results showed that as flow coefficient reduces, the decline in head coefficient gradually increases. Similarly, it was found that viscosity enhancement reduces the power coefficient. There was not any significant change in efficiency curves at low flow coefficients. In contrast, around design flow coefficient and at high flow coefficients, the hydraulic efficiency of the pump reduced by increasing viscosity of working fluid.

Keywords: regenerative pump, low specific speed, viscosity; polyacrylamide (PAM); performance curves.

Received September 07, 2016 Accepted December 07, 2017 